\title{
Positioning a focused Gaussian beam inside a refractive cylinder
}

\author{
Francisco Javier Salgado-Remacha ${ }^{\text {a,* }}$, José Antonio Sánchez-Martín ${ }^{\mathrm{b}}$, Eusebio Bernabeu ${ }^{\mathrm{b}}$ \\ a Universidad de Zaragoza, Department of Applied Physics, Zaragoza, Spain \\ ${ }^{\mathrm{b}}$ Universidad Complutense de Madrid, Applied Optics Complutense Group, Optics Department, Facultad de Ciencias Físicas, Ciudad Universitaria s.n., 28040 \\ Madrid, Spain
}

\section{A R T I C L E I N F O}

\section{Article history:}

Received 22 May 2013

Received in revised form

16 October 2013

Accepted 21 October 2013

Available online 6 November 2013

\section{Keywords:}

Diffraction

Beam propagation

Laser processing

Fiber-based sensors

\begin{abstract}
A B S T R A C T
We present in this work a study of the diffraction of a focused Gaussian beam by a microrefractive cylinder, and the dependence of the far field diffracted pattern with the location of the beam focal plane, relative to the center of the cylinder. A numerical study of the problem is carried out and validated with experimental verification. We center our attention on two parameters: the divergence and the number of peaks of the diffracted field. Both present a minimum for a particular position of the focal plane inside the refractive cylinder. This analysis can be used as a novel control technique for ablation of optical fibers and it can also be used as a criterion for the proper location of a focused laser beam inside an optical fiber with high accuracy. This criterion, based on the distribution of the far-field diffracted pattern, can be automated or used as a visual clue for a human operator.
\end{abstract}

(c) 2013 Elsevier Ltd. All rights reserved.

\section{Introduction}

The interaction of electromagnetic waves with refractive cylinders represents a canonical problem in optics and electromagnetism [1]. Depending on the relationship between the size of the cylinder diameter and the wavelength, several solutions have been proposed from geometrical optics [2] to the rigorous diffraction theory [3].

When the diameter of the cylinder is much larger than the wavelength, a geometrical optics-based approach is enough to predict the propagation of the field in the form of light rays refracted by cylinder lenses [4,5]. When we have cylinders or spheres with diameters below $100 \mu \mathrm{m}$, it can usually be assumed that the incident illumination field consists of plane waves. Under this approach, the classical works of Lorenz [6,7], Mie [8], and Debye [9] (conforming the Lorentz-Mie scattering theory) describe the propagation of light. Additionally, when the illumination is a focused Gaussian beam, the treatment of the problem is based on the separation of variables in a cylindrical coordinate system [10]. The large number of works published in the literature shows the interest in the study of these types of problems in many different fields, such as atmospheric optics [11], optical tweezers [12], and metrology [13]. Another application of these results is the use of electromagnetic radiation in the location and evaluation of infinite cylinders [14-16].

\footnotetext{
* Corresponding author. Tel.: + 34876553448 .

E-mail address: fjsalgado@unizar.es (F.J. Salgado-Remacha).
}

An alternative approach and a more recent point of view in this field have arisen from advances of the research in nano-optics. A plane wave that illuminates a refractive cylinder produces in its vicinity nanoscale fields in the shape of a jet, usually called "photonic nanojets" [17]. The main characteristics of nanojets are a very low beam waist (even smaller than the diffraction limit) and a very low divergence [17-20].

In our case, we are particularly interested in a specific application of that study. Laser micromachining of optic fibers represents a competitive and effective technique for optical sensor manufacturing [21,22]. To apply this technique, one must accurately know the location of the optical fiber (assumed as an infinite refractive cylinder) relative to the focal position of the laser. It should be noticed that, in this case, the beam width at the focal plane is much smaller than the cylinder diameter. Typical fiber diameters are around $125 \mu \mathrm{m}$ and the radii of focal spots are commonly $5 \mu \mathrm{m}$ or lower. Any misplacing of the focal point can produce critical damages in the optical fiber sensor, even its destruction.

Some works have been published that study the diffraction of a focused Gaussian beam by a refractive cylinder [23-25]. However, we are interested in a more flexible and visual method, and, if possible, one that can be automated. We propose in this paper to analyze the far-field shape of a highly-focused beam diffracted by an infinite refractive cylinder (representing an optical fiber), which provides a fast and easy visual method to place the fiber correctly with respect to the focal plane. As the first stage of our study, we show in this work a numerical analysis and a comparison with experimental data. As next sections will show, we obtain a visual criterion useful for our purpose. 


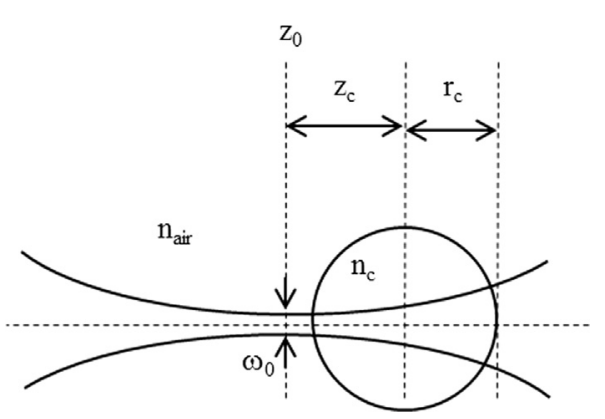

Fig. 1. Geometrical scheme of a Gaussian beam with beam waist $\omega_{0}$, travelling in air, impinging on a refractive cylinder with radius $r_{c}$ and refractive index $n_{c}$. The center of the cylinder is placed at $\left(z_{c}, x_{c}\right)$ relative to the location of the focusing point.

We perform an analysis of the system in Section 2, using a numerical algorithm for the simulation of light propagation. In Section 3, we perform an experimental analysis of the problem, showing the experimental setup. Both numerical and experimental results are compared, establishing a criterion. Finally, in Section 4 the final conclusions of this work are presented.

\section{Numerical approach}

As we have said, first we perform a numerical analysis of the system. We consider the optical fiber like an infinite refractive cylinder. In Fig. 1, the geometry of the system and the main parameters are shown. A focused Gaussian beam with spot radius $\omega_{0}$ travels through air. The refractive cylinder, with radius $r_{c}$ and refractive index $n_{c}$, is placed at a certain distance $z_{c}$ from the focal plane of the Gaussian beam.

In the simulations, we have used the beam propagation method (BPM) [26], a Fourier-transform-based Fresnel propagator, performed in Matlab. This algorithm simulates the propagation of an electromagnetic field under scalar approach, through a refractive index map [27]. It was originally developed for the simulations of optical waveguides [28], but it results were specially indicated for the simulation of any volumetric structure [29]. We then obtain the field at any point of the propagation map. In this work, we are interested in the intensity, calculated as $I(x, y, z)=U(x, y, z) U^{*}(x, y, z)$, where $U(x, y, z)$ and $U^{*}(x, y, z)$ denote the field and the conjugated field respectively at any point in space. It should be mentioned here that BPM takes into account only the propagating field. Thus, resonant modes and reflections at interfaces are neglected here. However, our analysis is still valid since it has been demonstrated that, due the features of our system, the dominant term in the diffracted pattern is the propagating term without reflections [17].

In our case, we first simulate the propagation of a focused Gaussian beam. Let us take the $Z$-axis as the propagation direction. Thus, the amplitude distribution of a Gaussian beam along the $X-Y$ plane can be written as

$U(x, y, z) \propto \exp \left[-\left(\frac{x}{\omega(z)}\right)^{2}-\left(\frac{y}{\omega(z)}\right)^{2}\right]$,

where $\omega(z)$ is a function defining the Gaussian beam width at any plane along the propagation axis, defined as [30]

$\omega(z)=\omega_{0} \sqrt{1+\left(\frac{z \lambda}{\pi \omega_{0}^{2}}\right)^{2}}$,

and $\omega_{0}$ is the minimum value of the Gaussian width.

In order to simulate the experimental conditions, we start from a Gaussian beam with wavelength $\lambda=454 \mathrm{~nm}$ and Gaussian width

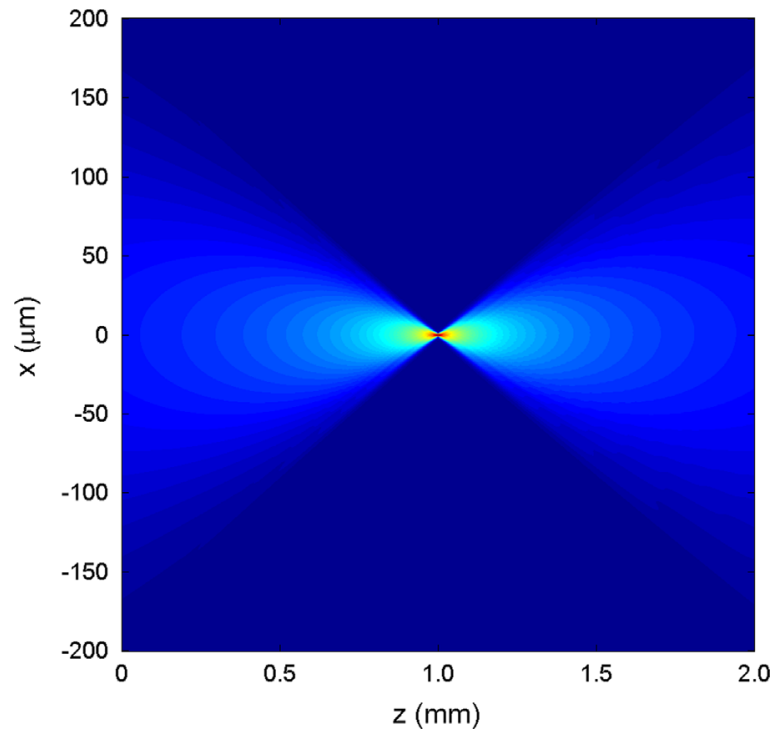

Fig. 2. Gaussian beam with beam radius $150 \mu \mathrm{m}$, focused by a thin spherical lens with $f=1 \mathrm{~mm}$ (logarithmical scale of intensities). Although the simulation was performed in a 3D space, only the plane at $y=0$ is shown.

$\omega_{0}=150 \mu \mathrm{m}$, placed at $z_{0}=0$. This beam is focused by a thin spherical lens, under paraxial approximation,

$L(x, y)=\exp \left[-j \frac{k}{2 f}\left(x^{2}+y^{2}\right)\right]$

where $j=\sqrt{-1}$ and $k=2 \pi / \lambda$ are the wave numbers, and $f$ is the focal distance of the lens, taken as $1 \mathrm{~mm}$.

Using this field as the initial illumination field for the BPM simulation through vacuum propagation, we obtain the intensity of a focused Gaussian beam, shown in Fig. 2.

Once we have a focused Gaussian beam, we introduce in the propagation space a refractive cylinder. In order to simulate an optical fiber, we choose the parameters of a common fiber, such as SMF-28 from Corning [31]. Thus, the diameter of the cylinder is given a value of $125 \mu \mathrm{m}$. Since the value of the refractive index of the cladding and the core are very close, we assume a constant refractive index of $n_{c}=1.46$ along the cylinder.

In order to maximize the resolution of the simulations, we divide the propagation in steps. First, we propagate the illumination field from the focusing lens to a plane close to the focal plane through air, with $n_{\text {air }}=1$. In our case, since $f=1 \mathrm{~mm}$, we propagate $800 \mu \mathrm{m}$ after the lens. Then, the last $X-Y$ plane of this propagation is taken as illumination field for the second propagation, with a total path of $400 \mu \mathrm{m}$ around the focal plane, placed at $z_{0}$. In this second step we introduce the refractive cylinder, with $n_{c}$, with its revolution axis along the $y$-axis and placed at $\left(x_{c}, z_{c}\right)$. The last $X-Y$ plane is used again as illumination field for the last step, in which we calculate the far field propagation using the discrete Fourier transform algorithm [32], serving us as a visual criterion.

We then obtain different resolutions for the propagation through free space (where low resolution is needed) and for the propagation in the vicinity of the focal plane. In Fig. 3, an example of the two last steps is shown. The center of the cylinder is placed in such a way that the beam does not impinge over the cylinder. Thus, the far field should have the form of a pure Gaussian function. Instead, we can appreciate in Fig. 3b some deviations from the pure Gaussian shape, subject to limitations in the angular spectrum due to the simulation restrictions.

When the focused light beam impinges over the cylinder, the diffraction pattern changes. For example, in Fig. 4 the propagation of an on-axis focused beam is shown. As can be appreciated in this case, the amount of intensity in higher diffracted orders grows. 
a

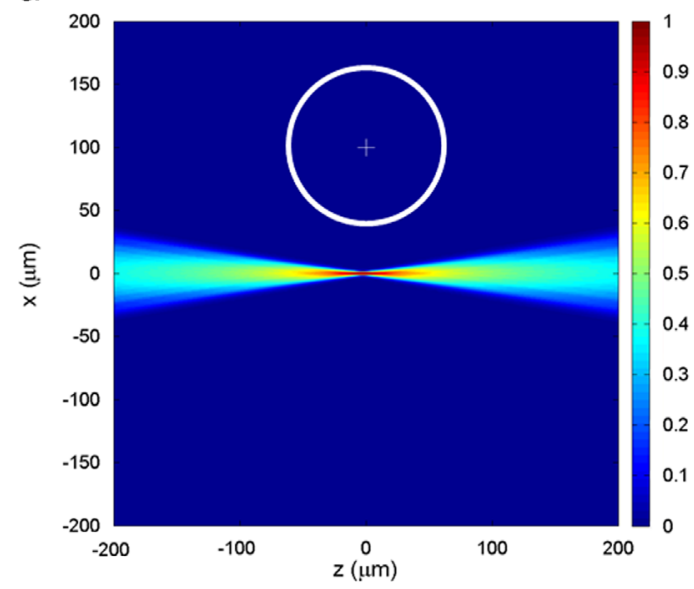

b

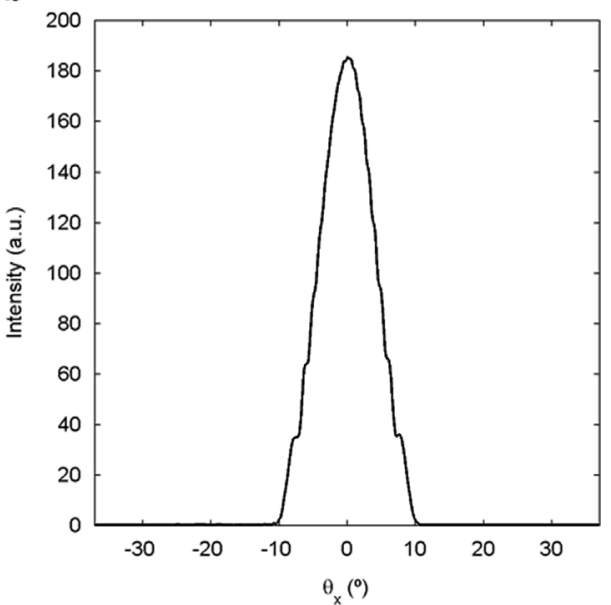

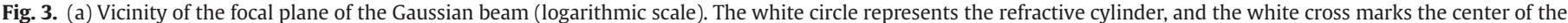
cylinder, placed at $x_{c}=100 \mu \mathrm{m}, z_{c}=z_{0}$ and (b) profile of the diffracted pattern of the last plane along the $Z$-axis in (a).

a

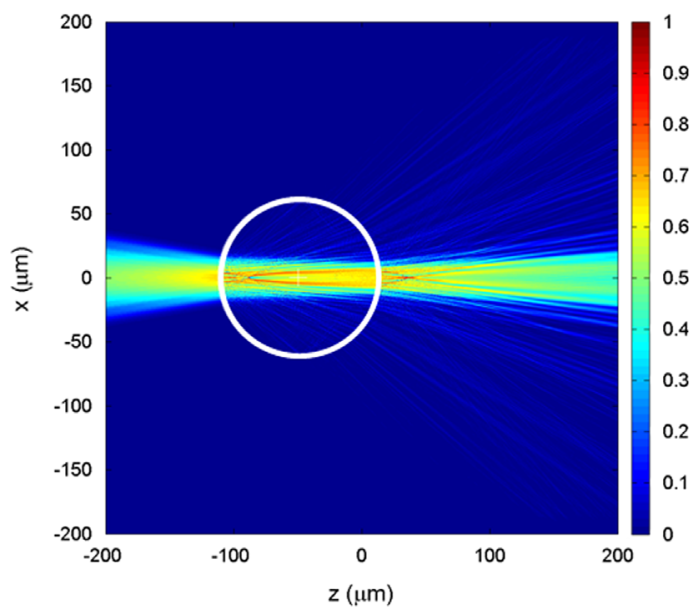

b

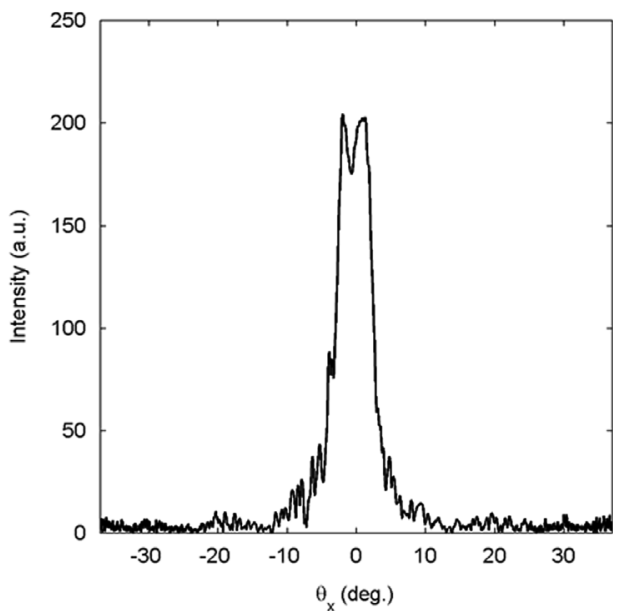

Fig. 4. Same as Fig. 3, with the cylinder placed at $x_{c}=0 \mu \mathrm{m}, z_{c}=-50 \mu \mathrm{m}$.

Placing the cylinder at a plane before the focal plane, and moving it to a plane behind the focal plane, we collect different diffraction patterns, shown in Fig. 5. Attending to the optical behavior in the far field, we have different regions. In the first region, with the center of the fiber placed before the focal plane, we have several diffraction orders out of the propagation direction. Along the central direction, an alternation of consecutive maxima and minima is appreciated, as the cylinder moves closer to the focal plane. The structure of the diffracted pattern is similar to the predictions of the Mie theory [33]. The next region corresponds to the cases in which the nominal focal plane falls inside the cylinder, which is interesting for optical fiber processing. In this region a narrow pattern appears with very low divergence similar to a Photonic nanojet [19]. Strictly, a photonic nanojet appears when a plane wave illumination is used. However, the most important characteristics of such a beam (very low divergence, high intensity) coincide with those of the observed pattern. Finally, the last region shows a behavior similar to the first one, with an alternation of the maxima and minima in the central direction, and an increasing number of diffraction orders as the cylinder moves away from the focal plane.

The appearance of a region with very low divergence and the variation of the number of peaks of the diffracted patterns can be used as guidance for the suitable location of the refractive cylinder relative to the focal plane of the beam. In the next section, we perform an experimental verification of these phenomena.

\section{Experimental validation}

In order to corroborate the simulations carried out in the last section, we perform an experimental analysis using the setup shown in Fig. 6.

An Argon tunable laser with different wavelengths, from 454 to $514 \mathrm{~nm}$ (model 543-AP-A01 from Melles Griot), is the light source. We work using $\lambda=454 \mathrm{~nm}$, as in the simulations. The output laser beam is coupled into an optical fiber, which also serves as a spatial filter. A collimating lens $(f=120 \mathrm{~mm})$ produces a beam with diameter $1 \mathrm{~mm}$. A focusing lens with $f=46 \mathrm{~mm}$ focuses the beam. Then, a third lens with $f=30 \mathrm{~mm}$ is placed only to obtain the far field of the diffracted pattern on the camera with the proper size. Finally, an iris is introduced in order to resize the beam diameter before the second lens.

The sensor is a CMOS camera by Imagingsource model DMK72BUC02. The pixel size is $2.2 \mu \mathrm{m} \times 2.2 \mu \mathrm{m}$ and the effective image area is $5.70 \mathrm{~mm}(H) \times 4.28 \mathrm{~mm}(V)$. The optical fiber is a standard SMF-28 by Corning. The fiber is placed without the 
acrylate protection in proximity of the focal plane, on $X-Y$ linear stages with micrometric precision.

Two hundred images were taken by placing the fiber at different distances, in steps of $1 \mu \mathrm{m}$. The initial position was carefully chosen, in order to place the point with $z_{c}=0$ at the middle of the measurement range. For any position the transversal location of the fiber must be corrected. Once we take all the diffracted patterns, we proceed as in the simulations. For any plane

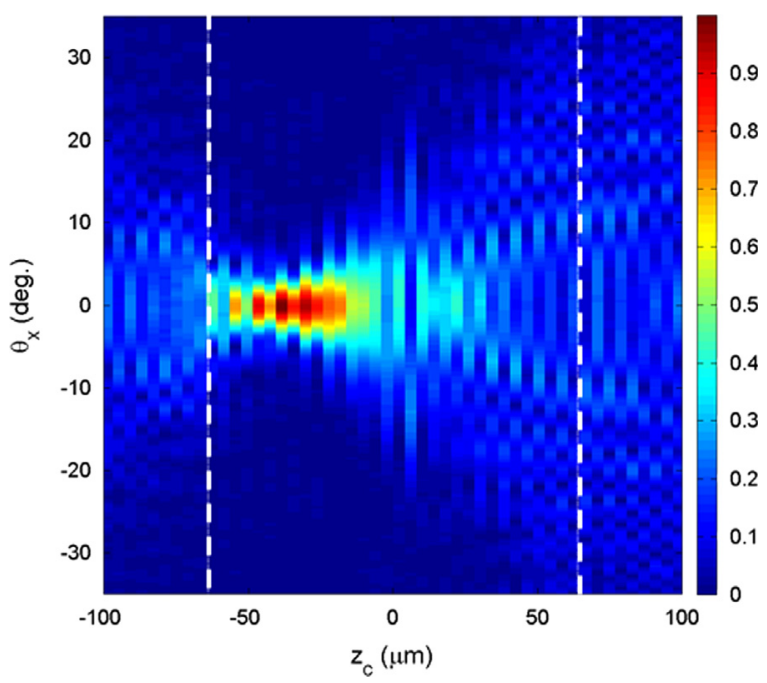

Fig. 5. Evolution of the far field pattern with the location of the cylinder for an onaxis focused beam with $\lambda=454 \mathrm{~nm}$. Vertical white dotted lines marks the interval in which the nominal focal plane is inside the cylinder. of measurement, we extracted a profile of the image along the central axis. We show these profiles in Fig. 7a. We can appreciate some differences, comparing with the simulations. First, we see off-axis propagation light in the regions where the focal plane is placed out of the fiber. We also observe a tightly rippled signal along the transversal coordinate. These differences can be due to resonant modes and internal reflections in the fiber. We must remember that BPM does not take into account back-reflections or counter-propagating modes. However, as we will see, for the results shown in this work the use of only propagating modes is enough [17]. In order to obtain a better signal, we smooth the data using a low-pass filter. The final diffracted patterns obtained are shown in Fig. $7 \mathrm{~b}$.

In order to compare the simulations with the experimental measurements, and thus to obtain a quantitative criterion, we perform two kinds of analyses: one of them based on the divergence of the diffracted beam, and the other one based on the number of peaks of the diffracted pattern for any location of the fiber around the focal plane.

We note that the diffracted beams do not correspond to a pure Gaussian beam, but to a more general distribution of energy. The common definitions of Gaussian width and divergence are defined for pure Gaussian modes. In this case, we make use of a generalized width, based on second-order moments of the diffracted patterns [30]. Let us assume an intensity distribution along one dimension, $I(x)$. First we define the "center of mass" of this distribution as

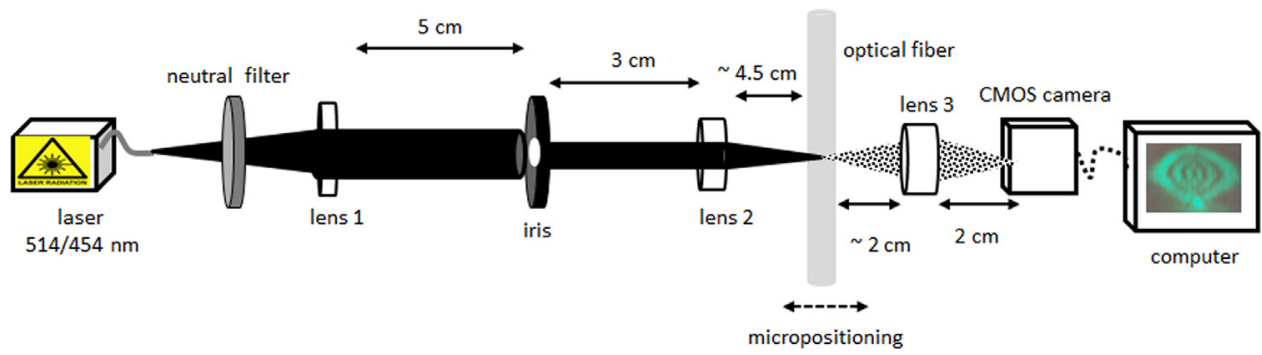

Fig. 6. Experimental setup.

a

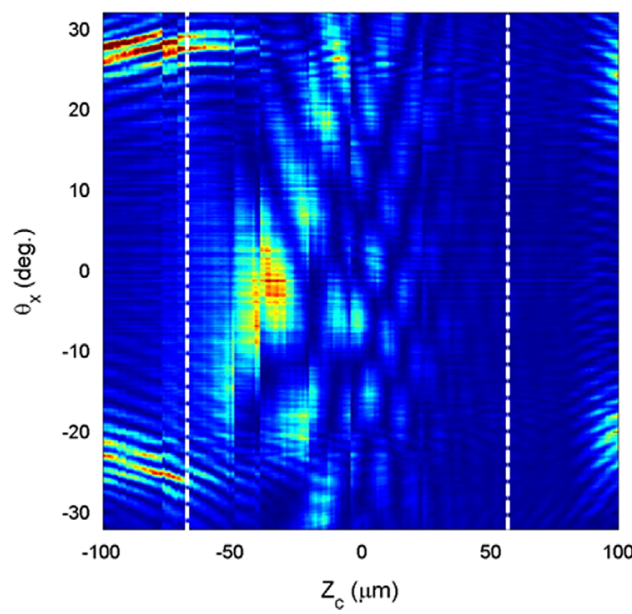

b

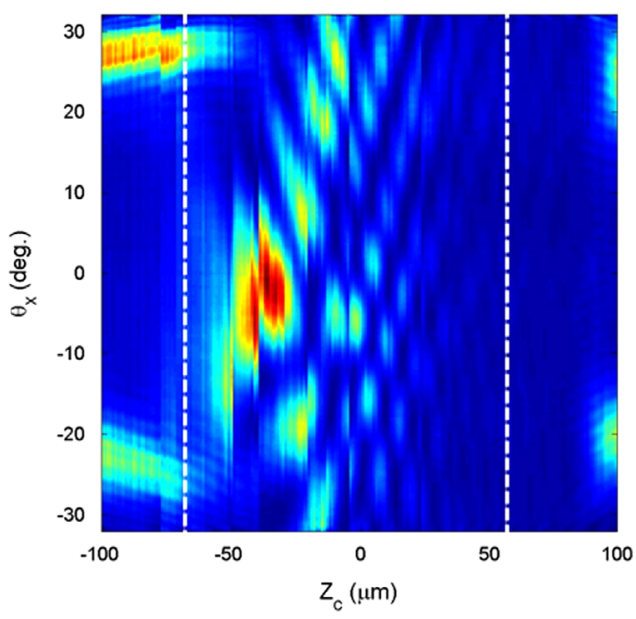

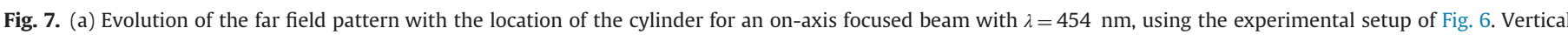

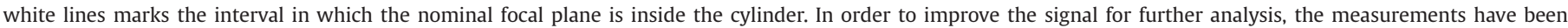
smoothed, shown in (b) 
a

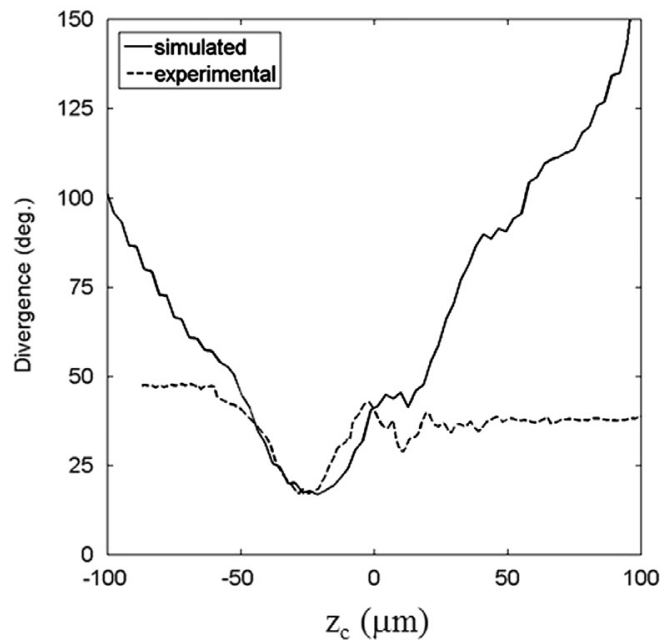

b

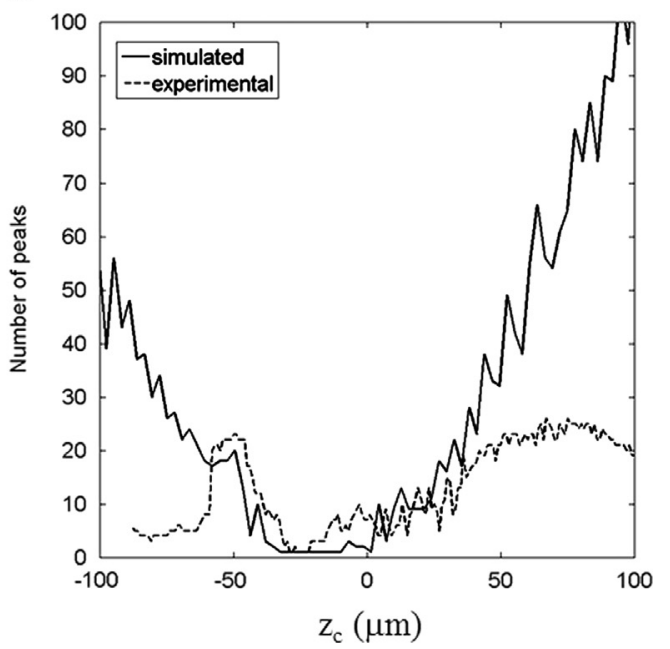

Fig. 8. (a) Divergence of the scattered beam, as the center of the cylinder moves along the $Z$-axis and (b) number of peaks at any plane along the propagation axis.

Then, the width of the diffracted pattern is defined as

$\omega_{x}(I)=2 \sqrt{\frac{\int_{-\infty}^{\infty} I(x)^{2}[x-x(I)]^{2} d x}{\int_{-\infty}^{\infty} I(x)^{2} d x}}$

Equivalently, the divergence $\Theta$ is defined as the width of the diffracted pattern at the Fourier plane. In other words, the longitudinal coordinate $x$ is substituted by the angular coordinate of the angular spectrum, $\theta$.

Applying Eq. (5) to the simulations in Fig. 5 and the filter measurements in Fig. $7 \mathrm{~b}$, we extract the divergence of the diffracted beam for any location of the fiber along the Z-axis, shown in Fig. 8a. It should be mentioned that the measurement of the divergence using a camera requires collecting most of the intensity on the chip of the camera. In our case, as can be seen in Fig. 7, this condition is not always reached. Thus, the measurement of the divergence of the experimental diffracted patterns is not always valid. However, our development is still valid for the case of the focal plane placed inside the cylinder, which is our main goal.

Attending to the divergence of the simulations, shown in Fig. 8a, we can see that the divergence decreases as the center of the fiber moves to the focal plane. A minimum divergence $\left(\theta_{\min }=17^{\circ}\right)$ appears when the fiber is placed at $z_{c}=-24 \mu \mathrm{m}$ (negative values mean locations at the left of the focal plane). After reaching this position, divergence increases again. The plot of the experimental divergence shows two planar zones, due to very high divergences (in other words, our camera-based method is not valid). However, in the region of low divergence, the experimental divergence follows a curve similar to that of the predicted divergence. The minimum appears at the same position, $z_{c}=-24 \mu \mathrm{m}$, and with the same value.

In order to improve our location criterion, we carry out another analysis based on the number of peaks along the diffracted pattern, as shown in Fig. 8b. As it can be seen, both measurements match again, showing the minimum number of peaks (only one peak) at the same position at which the divergence is minimum.

To summarize, we can use our analysis for positioning an optical fiber for laser ablation processing. It results in a very easy way to identify the position with minimum divergence or minimum number of peaks in the diffracted pattern. The identification of this point can be performed both visually and automatically by using a CCD array. Once we have identified this point, it is very easy to make a relative displacement from this point, being able to process the fiber with a good accuracy.

\section{Conclusions}

In this work we have performed an analysis of the diffracted pattern of a focused Gaussian beam by a transparent refractive infinite cylinder. This analysis has been carried out by means of numerical simulations using the BPM. At the same time, we have performed an experimental validation of the simulations. We note that there exists a relative location of the cylinder with respect to the focal plane, in which the divergence and the number of peaks are both minimal. The identification of this point is very easy, both visually and automatically. Thus, it is possible to place the focal plane inside the cylinder with great accuracy in terms of relative displacements. This method can be very useful for optical fiber processing using laser ablation.

\section{Acknowledgments}

This work has been partially supported by the IPT-0200002010-9 INNPACTO FORE Project of the Ministry of Science and Innovation of Spain. The authors also thank the comments and language editing of Dr. Jesús Martínez del Rincón, Prof. Dr. Agustín González-Cano, Elizabeth R Wright and Stephanie Smith.

\section{References}

[1] Born M, Wolf E. Principles of optics. 3rd. ed. Oxford, England: Pergamon Press Ltd; 1965 (Section 13.5).

[2] Casas J. Óptica. 7a ed. Spain: Universidad de Zaragoza; 1994.

[3] van de Hulst HC. Light scattering by small particles. New York, USA: John Wiley \& Sons; 1957.

[4] Winston R, Miñano JC, Benítez PG. Nonimaging optics. New York, USA: Elsevier-Academic Press; 2004.

[5] Mees L, Ren KF, Gréhan G, Gouesbet G. Scattering of a Gaussian beam by an infinite cylinder with arbitrary location and arbitrary orientation: numerical results. Appl Opt 1999;38:1867-76.

[6] Lorenz L. Lysbevaegelsen i og uden for en haf plane lysbölger belyst Kulge. Vidensk Selk Skr 1890;6:1-62.

[7] L. Lorenz, "Sur la lumière rèflèchie et rèfracèe par une sphère transparente," in Oeuvres Scientifiques de L. Lorenz, revues et annotèes par H. Valentiner (Librairie Lehmann et Stage, Copenhagen, 1898), pp. 405-529.

[8] Mie G. Beiträge zur Optik Trüber Medien, speziell Kolloidaler Meallösingen. Ann Phys 1908;25:377-452.

[9] Debye P. Der Lichtdruck auf Kugeln von Beliebigen Material. Ann Phys 1909;30:57-136.

[10] Barton JP. Internal and near-surface electromagnetic fields for an infinite cylinder illuminated by an arbitrary focused beam. J Opt Soc Am A 1999;16:160-6. 
[11] Xu L, Ding J, Cheng AYS. Scattering matrix of infrared radiation by ice finite circular cylinders. Appl Opt 2002;41:2333-48.

[12] Rockstuhl C, Herzig HP. Rigorous diffraction theory applied to the analysis of the optical force on elliptical nano- and micro-cylinders. J Opt A: Pure Appl Opt 2004;6:924-31.

[13] Doyle St. John W. Determination of the beam centroid of an obstructed focused Gaussian laser beam. Appl Opt 2009;48:4501-5.

[14] Gouesbet G, Maheu B, Gréhan G. Light scattering from a sphere arbitrarily located in a Gaussian beam, using a Bromwich formulation. J Opt Soc Am A 1988;5:1427-43.

[15] Nishiyama Y, Kurita S, Yamamoto I, Ishizuka Y, Watanabe T, Kobayashi D, et al. Diameter and refractive index of a cylindrical thread determined by scattered light pattern. Opt Rev 2001;8:90-4.

[16] Xu F, Ren KF, Cai X, Shen J. Extension of geometrical-optics approximation to on-axis Gaussian beam scattering. II. By a spherical particle with end-on incidence. Appl Opt 2006:45:5000-9.

[17] Itagi AV, Challener WA. Optics of photonic nanojets. J Opt Soc Am A 2005;22:2847-58.

[18] Lecler S, Takakura Y, Meyrueis P. Properties of a three-dimensional photonic jet. Opt Lett 2005;30:2641-3.

[19] Heifetz A, Kong S-C, Sahakian AV, Taflove A, Backman V. Photonic nanojets. J Comput Theor Nanosci 2009;6:1979-92.

[20] Geints YE, Panina EK, Zemlyanov AA. Control over parameters of photonic nanojets of dielectric microspheres. Opt Commun 2010;283:4775-81.

[21] Coelho J, Nespereira M, Silva C, Rebordao J. LOLS research in technology for the development and application of new fiber-based sensors. Sensors-Basel 2012;12:2654-66.

[22] Salgado-Remacha FJ, Sánchez-Martín JA, Bernabeu E, Procesado láser de fibras ópticas para el desarrollo de sensores de campo evanescente. Presented at the 2012 X Reunión Nacional de Óptica. Zaragoza, Spain; 2012.
[23] Casperson LW, Yeh C. Rayleigh-Debye scattering with focused laser beams. Appl Opt 1978;17:1637-43.

[24] Zimmermann E, Dändliker R, Souli N. Scattering of an off-axis Gaussian beam by a dielectric cylinder compared with a rigorous electromagnetic approach. J Opt Soc Am A 1995:12:398-403.

[25] Lock JA. Scattering of a diagonally incident focused Gaussian beam by an infinitely long homogeneous circular cylinder,. J Opt Soc Am A 1997;14: 640-52.

[26] Vanbrabant PJM, Beeckman J, Nayts K, James R, Fernandez FA. A finite element beam propagation method for simulation of liquid crystal devices. Opt Express 2009;17:10895-909.

[27] Salgado-Remacha FJ, Sanchez-Brea LM, Bernabeu E. Effect of fill-factor on the Talbot effect of diffraction gratings. J Eur Opt Soc-Rapid 2011;6:11055.

[28] Koshiba M, Tsuji Y, Hikari M. Finite element beam propagation method with perfectly matched layer boundary condition. IEEE Trans Magn 1999;35: 1482-5.

[29] Sirleto L, Iodice M, Della Corte GG, Rendina I. Digital optical switch based on amorphous silicon waveguide. Opt Laser Eng 2007;45:458-62.

[30] J. Alda, "Laser and Gaussian Beam Propagation and Transformation," in (Editor: Marcel Dekker) Encyclopedia of Optical Engineering ( Marcel Dekker, New York, USA, 2003), pp 999-1013.

[31] 〈http://www.corning.com/WorkArea/showcontent.aspx?id=41243〉.

[32] Schmidt JD. Numerical simulation of optical wave propagation with examples in Matlab. Washington, USA: SPIE Press; 2010 (Chapter 2).

[33] Berg MJ, Sorensen CM, Chakrabarti A. Explanation of the patterns in Mie theory. J Quant Spectrosc Radiat Trans 2010;111:782-94. 